\title{
Riscos ocupacionais inerentes aos profissionais do serviço de atendimento móvel de urgência
}

\section{Occupational risks inherent to mobile urgency service professionals}

\author{
Almir Rogério de Lima Teixeira' $\bullet$ Mayara Kelle Rodrigues de Carvalho ${ }^{2} \bullet$ Lúcio Lauro Leite dos Santos $^{3}$ \\ Jéssica Camelo Soares ${ }^{4}$ Andressa Maria de Sousa Moura ${ }^{5}$. Francileuza Ciríaco da Cruz ${ }^{6}$ \\ Braulio Vieira de Sousa Borges ${ }^{7}$ Isabel Cristina Cavalcante Carvalho Moreira ${ }^{8}$
}

\begin{abstract}
RESUMO
Objetivo: Identificar os riscos ocupacionais inerentes aos profissionais do serviço de atendimento móvel de urgência. Método: Estudo descritivo transversal, realizado com profissionais atuantes no Serviço de Atendimento Móvel de Urgência, na capital do Piauí, entre março e abril de 2019, com uso de questionário composto por perguntas fechadas, análise dos dados estatísticos descritivos e qui-quadrado com uso do software Statistical Package for the Social Sciences. Resultados: 91 profissionais atuantes, com predomínio do sexo masculino 59 (64,8\%); 43 (47,3\%) na faixa etária entre 45 a 60 anos; 3 I (34,I\%) ensino médio completo; 37 (40,7\%) técnicos em enfermagem; 56 (6I,5\%) mais de dez anos de atuação. 91 (100\%) apontaram a colisão automobilística e o sangue como os mais frequentes, o risco de fumaça de veículos com 89 (97,8\%), ruído de sirene $83(91,2 \%)$, levantamento de peso e o estresse, ambos com 86 (94,5\%). Conclusão: Predomínio do sexo masculino, adultos, com mais de oito anos de formação, técnicos em enfermagem, com longa data de exercício profissional e alta prevalência de riscos ocupacionais em diferentes categorias, com destaque para risco de colisão automobilística e agressões físicas, contaminação por sangue, levantamento de pesos, estresse situação. Descritores: Riscos Ocupacionais; Enfermagem em Emergência; Serviços Médicos de Emergência.
\end{abstract}

\begin{abstract}
Objective:To identify the occupational risks inherent to professionals of the mobile emergency care service. Method: Cross-sectional descriptive study, carried out with professionals working in the Mobile Emergency Service, in the capital of Piauí, between march and april 2019, using a questionnaire composed of closed questions, analysis of descriptive statistical data and chi-square with use of the Statistical Package for the Social Sciences software. Results: 91 active professionals, with a predominance of males 59 (64.8\%); 43 (47.3\%) in the age group between 45 and 60 years; 31 (34. I\%) completed high school; 37 (40.7\%) nursing technicians; 56 (61.5\%) more than ten years of experience. 91 (100\%) pointed to automobile collision and blood as the most frequent, the risk of vehicle smoke with $89(97.8 \%)$, siren noise 83 (91.2\%), weight lifting and stress, both with 86 (94.5\%). Conclusion: Predominance of males, adults, with more than eight years of training, nursing technicians, with a longstanding professional experience and high prevalence of occupational risks in different categories, with emphasis on the risk of automobile collision and physical aggression, contamination by blood, lifting weights, stress situation.

Descriptors: Occupational Risks; Emergency Nursing; Emergency Medical Services.
\end{abstract}

NOTA

I Acadêmico de Enfermagem pela Universidade Estadual do Piauí/Faculdade de Ciências Médicas (FACIME).Técnico de Enfermagem - Serviço de Atendimento Móvel de Urgência (SAMU) e Hospital Geral do Buenos Aires Drº Antônio Pedreira Martins.

2 Possui graduação em Enfermagem pela Universidade Estadual do Piauí.

3 Acadêmico de Enfermagem pela Universidade Estadual do Piauí/Faculdade de Ciências Médicas. Graduado em Ciências Biológicas pela Universidade Estadual do Maranhão/Centro de Estudos Superiores de Caxias.

4 Bacharel em enfermagem pela Universidade Estadual do Piauí - UESPI (2020). Atuação em projeto de pesquisa voltado para a segurança do paciente (PIBIC CNPq).Além disso, envolvimento em projetos de extensão em obstetrícia e análise de exames laboratoriais.

5 Possui graduação em Enfermagem pela Universidade Estadual do Piauí (2019).Atuação em projetos de pesquisa e extensão nas áreas de neonatologia, obstetrícia e segurança do paciente..

6 Possui graduação em Enfermagem pela Faculdade Integral Diferencial -FACID,(2016). Pós graduação em Gestão em Saúde pela Universidade Federal do Piaui/ Centro de Educação Aberta e a Distância-CEAD,(2019).Pós graduação em oncologia pela faculdade Unyleya,(2019).

7 Mestre em Enfermagem pela Universidade Federal do Piauí - Campus Ministro Petrônio Portella (2017). Especialista em Saúde Pública e da Família pelo Núcleo de Pós-graduação, Pesquisa e Extensão Faculdade KURIOS - FAK, Brasil. Email: braulitos89@hotmail.com

8 Possui graduação em Enfermagem pela Universidade Federal do Piauí (199I), graduação em Educação Física pela Universidade Federal do Piauí (I987) e mestrado em Enfermagem pela Universidade Federal do Piauí (2010). 


\section{INTRODUÇÃO}

Todos os anos, 2,34 milhões de pessoas morrem em consequência de doenças e acidentes advindos do trabaIho, sendo a grande maioria por doenças adquiridas durante a exposição laboral e os demais por acidentes que ocorrem durante a jornada de trabalho('). Dentre esses milhões de trabalhadores, estão os da área de saúde, e, dentre eles, estão inclusos os profissionais do Serviço de Atendimento Móvel de Urgência (SAMU), que compõe a rede de atenção das urgências e emergências, criado com o objetivo de disponibilizar atendimento imediato e transportar adequadamente, de forma resolutiva e rápida indivíduos em situação de urgência, e é normatizado pela Portaria $\mathrm{n}^{\circ} 1.010$, de maio de 2012, do Ministério da Saúde ${ }^{(2)}$.

Autores destacam que os profissionais atuantes no SAMU são suscetíveis a diversos riscos ocupacionais, assim como têm conhecimento dos fatores de risco envolvidos durante o seu labor, considerando a sobrecarga de trabalho aliada às falhas na estrutura organizacional, fatores esses determinantes para potencializar os riscos ocupacionais $^{(3)}$.

A identificação precoce dos riscos ocupacionais a que esses profissionais estão expostos exerce fundamental importância na criação de medidas de prevenção e no controle dos riscos, assim como possibilita agir previamente sobre os acidentes e as doenças relacionadas ao trabalho ${ }^{(4)}$.Ainda, o conceito de gerenciamento de riscos no departamento de emergência contém um significado mais amplo, de modo que é conhecido como evento ou situação repentina que pode acontecer em um futuro incerto, com alguns impactos negativos ou positivos que podem ser chamados de ameaça ou oportunidade, respectivamente ${ }^{(5)}$.

Assim, entende-se por riscos ocupacionais as condições de vulnerabilidades capazes de causar danos à saúde, aos quais os trabalhadores se expõem durante a execução de suas atividades, seja por meio dos acidentes, das doenças ou de agravos de natureza psicológica da pessoa exposta ${ }^{(3)}$. Segundo o Ministério da Saúde do Brasil, os riscos mais frequentes nos ambientes de trabalho, por categoria, são: físicos (ruídos, temperaturas extremas, iluminação, radiação ionizante e não ionizante), químicos (substâncias químicas que podem estar presentes nos ambientes de trabalho na forma de poeiras, fumos, névoas, neblinas, gases ou vapores), mecânicos (máquinas com partes móveis não protegidas; calandras e cilindros; guiIhotinas; prensas e o uso de instrumentos cortantes ou perfurantes etc.), biológicos (micro-organismos, como bactérias, fungos, protozoários, vírus, entre outros; e animais peçonhentos, como cobras, escorpiões, aranhas), e psicossociais (jornadas de trabalho longas, esforços físicos exagerados com posturas forçadas e carregamento de peso, ritmo acelerado, trabalho repetitivo e monótono; trabalho em turnos e noturno; desemprego; vínculos precários ou ausência de vínculo trabalhista) ${ }^{(6)}$.

Dentre essas, há uma subcategoria que se destaca, compreendendo os ergonômicos, denominada de riscos psicossociais, como a tensão e a supervisão constante, as horas extras, a carga horária excessiva, as escalas noturnas, as cobranças de produtividade, a imposição de tarefas pelos superiores, os assédios morais etc. ${ }^{(7)}$. Estudo realizado nos serviços médicos de emergência no Irã apontam elevada prevalência de violência no local de trabalho entre os funcionários, e recomenda que mais estudos devam ser conduzidos para determinar as causas subjacentes da violência no local de trabalho dos funcionários ${ }^{(8)}$.

Os enfermeiros de emergência estão expostos a eventos traumáticos e estressores de rotina, os quais podem levar ao desenvolvimento da sintomatologia do transtorno de estresse pós-traumático( ${ }^{(9)}$. Dessa forma, destaca-se que, entre os riscos psicossociais, como a exaustão no trabalho, o estresse e a insatisfação estão ligados à qualidade do atendimento, aos resultados dos pacientes e à retenção de funcionários, repercutindo diretamente em assistência de qualidade ${ }^{(10)}$.

Urge a necessidade de debater acerca dos riscos ocupacionais aos quais os profissionais atuantes no SAMU se encontram expostos enquanto trabalham, e, baseado nos dados resultantes, levantar reflexões e campo de enfrentamento de riscos em busca de estratégias que possam atenuar a ocorrência de incidentes e de enfermidades que podem desencadear ao trabalhador, o que, consequentemente, repercutirá na qualidade da assistência à população. Nesse âmbito, o estudo propôs identificar os riscos ocupacionais inerentes aos profissionais do Serviço de Atendimento Móvel de Urgência.

\section{MÉTODO}

Estudo transversal do tipo descritivo de abordagem quantitativa, realizado com profissionais atuantes no Serviço de Atendimento Móvel de Urgência, na capital do Piauí, entre março e abril de 2019.

Entre os participantes, foram inclusos apenas os profissionais que trabalham prestando assistência direta aos pacientes nas ambulâncias e motolâncias. Foram excluídos os que estavam afastados do serviço por licença-prêmio, licença-maternidade, férias, tratamento de saúde e outros motivos. Os 169 servidores estão assim distribuídos: 21 médicos, 20 enfermeiros, 69 técnicos em enfermagem e 59 condutores socorristas. A amostra final foi de 91 participantes, sendo II médicos, II enfermeiros, 37 técnicos em enfermagem e 32 condutores. Foi considerado nível de confiança de $95 \%$, erro máximo de $5 \%$ e prevalência presumida e complementar de $50 \%$. 
Assim, a amostragem foi do tipo não probabilística estratificada por conveniência.

Para coleta de dados, utilizou-se um questionário semiestruturado contendo perguntas fechadas quanto a aspectos sociodemográficos (sexo, faixa etária, profissão, nível de escolaridade e tempo de atividade ocupacional) e riscos ocupacionais (acidentais, biológicos, físicos, químicos, ergonômicos e psicossociais). A coleta de dados ocorreu no local de atuação dos profissionais, com dia e turno previamente agendados, respeitando as escalas de serviço e a disponibilidade dos participantes.

Os dados foram organizados e digitados duplamente no Microsoft Excel, em planilha eletrônica, e, após validação, foram exportados para o software IBM $®$ Statistical Package for the Social Sciences (SPSS) 21.0. Para análise das variáveis, utilizou-se frequências relativa e absoluta; ainda, empregou-se o teste qui-quadrado, sendo considerada a significância estatística em valor de $\mathrm{p}<0,005$.
A pesquisa obedeceu a todos os preceitos éticos da Resolução $n^{\circ} 466$, de 12 de dezembro de 2012, sendo aprovado em março de 2019, com o número de CAAE: 08I31819.5.0000.5209 e com o Parecer $n^{\circ} 3.234 .101$. Destaca-se que os participantes foram orientados quanto aos objetivos do estudo e, após concordarem, assinaram o Termo de Consentimento Livre e Esclarecido.

\section{RESULTADOS}

Dos 91 profissionais, houve predominância do sexo masculino 59 (64,8\%), faixa etária de 45 a 60 anos 43 (47,2\%), 37 (40,7\%) técnicos em enfermagem, 3 I (34,0\%) com ensino médio completo e $56(61,5 \%)$ com mais de dez anos de atividade ocupacional (Tabela I).

Quanto aos riscos ocupacionais, 91 (100\%) relataram risco acidental decorrente da colisão automobilística e 88 (96,7\%) riscos de agressões físicas; 91 (100\%) risco de contaminação biológica por sangue; 83 (91,2\%) ruídos de sirene (Tabela 2).

TABELA 1 - Perfil referente aos profissionais do Serviço de Atendimento Móvel de Urgência ( $n=91$ ). Teresina, Piauí, Brasil, 2019

\begin{tabular}{|c|c|c|c|}
\hline Variáveis & $\mathbf{N}$ & $\%$ & p-valor \\
\hline \multicolumn{4}{|l|}{ Sexo } \\
\hline Masculino & 59 & 64,8 & 0,005 \\
\hline Feminino & 32 & 35,2 & \\
\hline \multicolumn{4}{|l|}{ Faixa etária (anos) } \\
\hline$<25$ & - & - & $<0,001$ \\
\hline $25-35$ & 14 & 15,4 & \\
\hline $35-45$ & 25 & 27,5 & \\
\hline $45-60$ & 43 & 47,2 & \\
\hline$>60$ & 9 & 9,9 & \\
\hline \multicolumn{4}{|l|}{ Profissão } \\
\hline Condutor & 32 & 35,1 & $<0,001$ \\
\hline Enfermeiro & 11 & 12,1 & \\
\hline Técnico em Enfermagem & 37 & 40,7 & \\
\hline Médico & 11 & 12,1 & \\
\hline \multicolumn{4}{|l|}{ Nível de escolaridade } \\
\hline Ensino Fundamental incompleto & 2 & 2,2 & $<0,001$ \\
\hline Ensino Fundamental completo & 3 & 3,3 & \\
\hline Ensino Médio incompleto & 4 & 4,4 & \\
\hline Ensino Médio completo & 31 & 34,0 & \\
\hline Superior incompleto & 11 & 12,1 & \\
\hline Superior completo & 14 & 15,4 & \\
\hline Pós-graduação & 26 & 28,6 & \\
\hline \multicolumn{4}{|l|}{ Tempo de atividade ocupacional } \\
\hline$<1$ & 3 & 3,3 & $<0,001$ \\
\hline $1-3$ anos & 5 & 5,5 & \\
\hline $3-5$ anos & 5 & 5,5 & \\
\hline $5-10$ anos & 22 & 24,2 & \\
\hline$>10$ anos & 56 & 61,5 & \\
\hline
\end{tabular}

Legenda: N=número; \% percentual; * $p$ - Teste Qui-quadrado.

Fonte: Dados da pesquisa, 2019. 
No tocante aos riscos químicos, $89(97,8 \%)$ informaram fumaça de veículos e apenas 40 (44,0\%) citaram medicamentos; 86 (94,5\%) apontaram como risco ergonômico o levantamento de pesos. Já em relação aos riscos psicossociais, 86 (94,5\%) apontam estresse situacional, e 34 (37,4\%) acusam a jornada de trabalho prolongada (Tabela 2 ).

\section{DISCUSSÃO}

Os achados deste estudo apontam para altos riscos ocupacionais de diferentes categorias. Estudos em âmbito internacional corroboram esses achados ${ }^{(1-13)}$. Pesquisa realizada no cenário nacional conclui que a gestão participativa e o aperfeiçoamento profissional poderão minimizar a exposição dos servidores dessas áreas a diferentes tipos de riscos ocupacionais ${ }^{(14)}$. Diante desse aspecto, faz-se necessário identificar a natureza dos riscos ocupacionais nos diferentes níveis de atuação dos profissionais, bem como traçar estratégias intervencionistas de

TABELA 2 - Riscos ocupacionais entre os profissionais do Serviço de Atendimento Móvel de Urgência ( $n=91$ ). Teresina, Piauí, Brasil, 2019

\begin{tabular}{|c|c|c|c|}
\hline Variáveis & $\mathbf{N}$ & $\%$ & p-valor* \\
\hline \multicolumn{4}{|l|}{ Riscos Acidentais } \\
\hline Colisão automobilística & 91 & 100,0 & $<0,001$ \\
\hline Risco de choque elétrico & 44 & 48,4 & \\
\hline Riscos de agressões físicas & 88 & 96,7 & \\
\hline \multicolumn{4}{|l|}{ Riscos Biológicos } \\
\hline Sangue & 91 & 100,0 & $<0,001$ \\
\hline Líquido cefalorraquidiano & 23 & 25,3 & \\
\hline Vômitos & 89 & 97,8 & \\
\hline Salivas & 83 & 91,2 & \\
\hline Secreções nasais & 73 & 80,2 & \\
\hline Líquidos pleurais & 20 & 22,0 & \\
\hline Líquidos amnióticos & 72 & 79,1 & \\
\hline \multicolumn{4}{|l|}{ Riscos Físicos } \\
\hline Exposição a altas temperaturas climáticas & 82 & 90,1 & $<0,001$ \\
\hline Radiações ionizantes e não ionizantes & 36 & 39,6 & \\
\hline Ruídos de sirene & 83 & 91,2 & \\
\hline Vibrações & 63 & 69,2 & \\
\hline \multicolumn{4}{|l|}{ Riscos Químicos } \\
\hline Hipoclorito de sódio & 78 & 85,7 & $<0,001$ \\
\hline Álcool & 64 & 70,3 & \\
\hline Medicamentos & 40 & 44,0 & \\
\hline Poeira & 71 & 78,0 & \\
\hline Fumaça de veículos & 89 & 97,8 & \\
\hline \multicolumn{4}{|l|}{ Riscos Ergonômicos } \\
\hline Levantamento de pesos & 86 & 94,5 & 0,461 \\
\hline Repetitividade de tarefas e peso & 73 & 80,2 & \\
\hline Postura inadequada habitual & 73 & 80,2 & \\
\hline Intensa rotina & 67 & 73,6 & \\
\hline \multicolumn{4}{|l|}{ Riscos Psicossociais } \\
\hline Estresse Situação & 86 & 94,5 & $<0,001$ \\
\hline Carga de trabalho excessiva & 36 & 39,6 & \\
\hline Agressões verbais & 84 & 92,3 & \\
\hline Jornada de trabalho prolongada & 34 & 37,4 & \\
\hline Jornadas noturnas & 54 & 59,3 & \\
\hline Elevada tensão ambiental frequente & 55 & 60,4 & \\
\hline Prejuízos na inter-relação com a chefia & 37 & 40,7 & \\
\hline Prejuízos na interação com colegas de trabalho & 38 & 41,8 & \\
\hline
\end{tabular}

Legenda: N=número; \% percentual; * $p$ - Teste Qui-quadrado.

Fonte: Dados da pesquisa, 2019. 
ações proativas, a fim de minimizar os riscos à saúde do trabalhador e da população.

Neste estudo, houve predominância de adultos do sexo masculino, com mais de oito anos de estudos. Pesquisa realizada nos serviços médicos de emergência asiáticos também relevou tais achados ${ }^{(15)}$. Em âmbito nacional, estudos evidenciam o contrário quanto ao sexo ${ }^{(14)}$. Assim, enfatiza-se a importância da abordagem do sexo e da idade na elaboração de políticas de saúde do trabalhador, que visem contextualizar de maneira ampla e singular as peculiaridades do homem no seu contexto de trabalho e dos riscos ocupacionais referentes.

Em relação às diferentes categorias de riscos ocupacionais, a colisão automobilística e as agressões físicas prevaleceram neste estudo, o que vai ao encontro da estatística registrada na literatura em âmbito global, pois sabe-se que o atendimento pré-hospitalar móvel é marcado pela frequente ocorrência de acidentes automobilísticos, e que também não são incomuns as agressões físicas envolvendo profissionais de saúde que trabalham nas ruas ${ }^{(16)}$.Autores enfatizam sobre a necessidade de estratégias para prevenir a violência no local de trabalho, que devem ser adaptadas às práticas locais e efetivamente implementadas ${ }^{(15)}$.

O risco biológico por sangue teve destaque neste estudo, citado pelos participantes. Pesquisa realizada na República da Coreia, ao avaliar a exposição ocupacional ao risco de infecção e o uso de equipamentos de proteção individual por pessoal médico de emergência, obteve risco ocupacional por fluidos corporais e sangue $(40,2 \%)$, sendo a lesão por agulha o tipo de risco mais frequente $(17,6 \%)$ (13). Autores apontam que os trabalhadores de serviços médicos de emergência correm risco de exposição a patógenos transmitidos pelo sangue e que são frequentemente expostos a sangue e fluidos corporais por lesões percutâneas ${ }^{(12)}$. Assim, é necessária a educação permanente entre os profissionais de saúde de serviços de atendimento de emergência para o uso correto de equipamentos de proteção individual (EPI), assim como o provimento de insumos indispensáveis para o atendimento seguro e eficaz aos envolvidos no cenário assistencial.

O ruído de sirene e a exposição a altas temperaturas climáticas foram os riscos físicos mais prevalentes, assemelhando-se ao encontrado em outros dois estudos sobre riscos ocupacionais ${ }^{(17-18)}$. $O$ uso de protetores auriculares deve fazer parte dos equipamentos de proteção dos trabalhadores de serviços de emergência, a fim de evitar transtornos ocupacionais e agravos à saúde auricular, com repercussão no sistema biológico. Dessa forma, a disponibilização de insumos de proteção auricular deve ser pauta das políticas de gestão local com respeito às singularidades e às normas de segurança dos trabalhadores.
Para os riscos químicos, a exposição à fumaça de veículos prevaleceu sobre os demais. Em experiências realizadas nos Estados Unidos da América com ratos em laboratório, com o objetivo de investigar os efeitos da exposição prolongada às emissões dos escapes de veículos, os pesquisadores concluíram que resultou em alterações das funções comportamentais e cognitivas, tais como ansiedade, depressão e memória prejudicada ${ }^{(19)}$. Políticas de saúde pública e de saúde ambiental devem articular estratégias de prevenção e redução de danos à saúde dos trabalhadores e da sociedade, bem como de proteção ao meio ambiente.

Neste estudo, o levantamento de peso é o risco ergonômico notório entre os participantes.Autores apontam que como consequência disso, os socorristas desenvolvem diversos problemas de saúde, sendo mais comuns os distúrbios osteomusculares, como dores e lesões nas regiões da coluna, nos membros superiores e nos inferiores. O risco de adoecimento aumenta quando se somam o peso e as situações adversas ergonomicamente inapropriadas encontradas no campo de trabalho desses profissionais ${ }^{(20)}$. Sugere-se que, durante os turnos de trabalhos, tenham-se momentos de práticas integrativas complementares, como exercícios de alongamentos, técnicas de relaxamento muscular e massagens para maior rendimento na jornada de trabalho, bem como redução de danos na saúde do sistema musculoesquelético.

No que tange ao risco psicossocial, o estresse prevaleceu neste estudo.Autores afirmam que os enfermeiros que trabalham nos departamentos de emergência testemunham acidentes desagradáveis e estão mais expostos ao estresse e à violência do que outros profissionais ${ }^{(1)}$. Estudo realizado no Irã conclui que mais de $60 \%$ dos enfermeiros de emergência apresentaram níveis moderados de desgaste no trabalho, estresse e insatisfação(10). Urge a necessidade de terapias individuais ou coletivas entre os trabalhadores atuantes nos serviços de urgência, a fim de reduzir o estresse ocupacional e potencializar estratégias de prevenção e promoção da saúde mental.

Destaca-se, neste estudo, que agressões verbais fazem parte dos riscos dos profissionais de saúde. Pesquisa que teve como objetivo avaliar a prevalência de tipos de violência no local de trabalho, contra funcionários de serviços médicos de emergência no Irã, encontrou a prevalência de violências física, verbal e cultural no local de trabalho entre os funcionários de $36,39 \%$, $73,13 \%$ e $16,51 \%$, respectivamente. Ainda, recomenda o treinamento de métodos de prevenção à violência e a atribuição de regras de proteção nacionais ${ }^{(8)}$. Autores concluem que o treinamento e a aplicação da massagem terapêutica podem servir como métodos eficazes para a redução do estresse ocupacional em centros médicos de emergência ${ }^{(21)}$. 
Nessa perspectiva, autores destacam que há inúmeras situação que podem atrapalhar o atendimento do SAMU, como locais imprevisíveis, materiais e recursos humanos limitados e apropriados para garantir a assistência de saúde a vítima, podendo afetar diretamente a qualidade do atendimento prestado(22).

Como apontam os achados, todos as categorias de riscos apresentam alta prevalência, esse fato exige que os profissionais busquem cada vez mais qualificação profissional, sobretudo no que diz respeito às medidas de prevenção e de segurança no trabalho.

\section{CONCLUSÃO}

Constata-se alta prevalência de riscos ocupacionais em diferentes categorias entre profissionais do Serviço de Atendimento Móvel de Urgência, com destaque para risco de colisão automobilística e agressões físicas, contaminação por sangue, ruídos de sirene, fumaças de veículos, levantamento de peso excessivo, estresse, situação de agressões verbais.Ainda, predomínio de profissionais adultos do sexo masculino, com mais de oito anos de formação, predomínio dos técnicos em enfermagem, com longa experiência profissional.

Quanto às limitações deste estudo, resume-se ao fato de não trabalhar $\circ$ universo populacional tendo apenas uma amostragem, que, por conveniência, foi do tipo estratificada entre categorias profissionais. Ainda assim, recomenda-se novos estudos para maior aprofundamento da temática, e práticas em saúde com enfoque nas medidas de prevenção e segurança do trabalhador baseadas nas melhores evidências científicas no âmbito internacional, nacional e local. 


\section{REFERÊNCIAS}

I. Hämäläinen P, Takala J, Kiat TB. Global Estimates of Occupational Accidents and Work-related Illnesses 2017. World Congress on Safety and Health at Work 2017,3-4 September 2017 Singapore. Workplace Safety and Health Institute. Disponível em: http://www.icohweb.org/site/images/news/ pdf/Report\%20Global\%20Estimates\%20of\%200ccupational\%20Accidents\%20and\%20Work-related\%20IIInesses\%202017\%20revl.pdf

2. Brasil. Ministério da Saúde. Secretaria de Atenção à Saúde.

Departamento de Atenção Especializada. Manual instrutivo da Rede de Atenção às Urgências e Emergências no Sistema Único de Saúde (SUS). $2^{a}$ ed. Brasília: Editora do Ministério da Saúde, 2013.84 p.

3. Nascimento MO, Araújo GF. Riscos Ocupacionais dos Profissionais de Enfermagem atuante no SAMU 192. Id on Line Revista Multidisciplinar e de Psicologia, Jaboatão dos Guararapes-PE [internet]. 2017 [acesso em 6 jul 2020]; I0(33): 2 I2-223. Disponível em: https://idonline.emnuvens.com.br/ id/article/view/6|4/864

4. Gonçalves DF, Giotto AC. Prevalência de acidentes do trabalho com profissionais da enfermagem do sistema de saúde. Rev Inic Cient e Ext. Chácaras Anhaguera Valparaiso de Goiás [internet]. 2018 [acesso em 2 jul 2020]; 2(2): I09I | 7. Disponível em: https://revistasfacesa.senaaires.com.br/ index.php/iniciacao-cientifica/article/view/ I55/ I I0

5. Tavirani MR, Beigvand HH.A Review of Various Methods of Management of Risk in the Field of Emergency Medicine. Open Access Maced J Med Sci [internet]. 2019 [acesso em 2 jul 2020]; 7(23): 4I79-4I87. Disponível em: https://www. ncbi.nlm.nih.gov/pmc/articles/PMC706 I 389/pdf/OAMJMS-7-4I79.pdf

6. Brasil. Ministério da Saúde. Secretaria de Atenção à Saúde. Secretaria de Vigilância em Saúde. Saúde do trabalhador e da trabalhadora [recurso eletrônico]. Cadernos de Atenção Básica, n. 4I. Brasília: Editora do Ministério da Saúde, 2018.

7. Abiramalakshmi A, Shree KC R. Systematic measurement of occupational hazards among healthcare workers. International Journal of Physiology, Nutrition and Physical Education, Nova Délhi-Índia [internet]. 2018 [acesso em 6 jul 2020]; 3(2): 560-563. Disponível em: http://www.journalofsports.com/pdf/2018/vol3issue2/PartM/3-2-138-752.pdf

8. Sahebi A, Jahangiri K, Sohrabizadeh S, Golitaleb M. Prevalence of Workplace Violence Types against Personnel of Emergency Medical Services in Iran:A Systematic Review and Meta-Analysis. Iran J Psychiatry [internet]. 2019 [acesso em 2 jul 2020]; I4(4): 325-334. Disponível em: https://www.ncbi.nlm. nih.gov/pmc/articles/PMC7007507/pdf/IJPS- |4-325.pdf

9. Campillo-Cruz M, González-Gutiérrez JL, Ardoy-Cuadros J, Fernández-Muñoz JJ. The Development and Exploratory Psychometric Properties of the Traumatic and Routine Stressors Scale on Emergency Nurses (TRSS-EN). Int J En- viron Res Public Health [internet]. 2020 [acesso em 6 jul 2020]; 17(6): 1963. Disponível em: https://www.ncbi.nlm. nih.gov/pmc/articles/PMC7|43067/pdf/ijerph-17-01963.pdf

10. Tavakoli N, Shaker SH, Soltani S, Abbasi M, Amini M, Tahmasebi A et al. Job Burnout, Stress, and Satisfaction among Emergency Nursing Staff after Health System Transformation Plan in Iran. Emerg (Tehran) [internet]. 2018 [acesso em 6 jul 2020]; 6(I): e4I. Disponível em: https://www.ncbi.nlm.nih.gov/pmc/articles/PMC6289 I6I/ pdf/emerg-6-e4l.pdf

II. Hosseininejad SM, Jahanian F, Elyasi F, Mokhtari H, Koulaei ME, Pashaei SM. The prevalence of post-traumatic stress disorder among emergency nurses: a cross sectional study in northern Iran. Biomedicine (Taipei) [internet]. 2019 [acesso em 6 jul 2020]; 9(3): 19. Disponível em: https://www.ncbi.nlm.nih.gov/pmc/articles/PMC6289/6I/pdf/ emerg-6-e4l.pdf

12. Alhazmi RA, Parker RD,Wen S. Standard Precautions Among Emergency Medical Services in Urban and Rural Areas. Workplace Health Saf [internet]. 2020 [acesso em 6 jul 2020]; 68(2): 73-80. Disponível em: https://www.ncbi.nlm. nih.gov/pmc/articles/PMC704|279/pdf/nihms-1065237.pdf

13. Oh HS, Uhm D. Occupational exposure to infection risk and use of personal protective equipment by emergency medical personnel in the Republic of Korea. Am J Infect Control [internet]. 2016[acesso em 6 jul 2020]44(6): 64765I. Disponível em: https://www.ncbi.nlm.nih.gov/pmc/articles/PMC7I 32644/pdf/main.pdf

14. Leite HDCS, Carvalho MTR, Cariman SLS, Araujo ERM, Silva NC, Carvalho AO, et al. Riscos ocupacional entre profissionais de saúde do Serviço de Atendimento Móvel de Urgência-SAMU. Enferm. Foco [internet]. 2016 [acesso em 6 jul 2020]; 7(3/4): 31-35. Disponível em: http://revista.cofen.gov.br/index.php/enfermagem/article/view/9/2/342

15. Wang PY, Fang PH, Wu CL, Hsu HC, Lin CH. Workplace Violence in Asian Emergency Medical Services:A Pilot Study. Int J Environ Res Public Health [internet]. 2019 [acesso em 6 jul 2020]; I6(20): 3936. Disponível em: https:// www.ncbi.nlm.nih.gov/pmc/articles/PMC6843 I I 9/pdf/ijerph-16-03936.pdf

16. Bozorgi F, Laali A, Khademloo M, Ehteshami S, Assadi T, Pashaie SM, et al. Incidence and Risk Factors of Workplace Violence against Pre Hospital Staffs in Mazandaran; North of Iran. Int J Med Invest. Novi Sad, Serbia [internet]. 2018 [acesso em 6 jul 2020]; 7(4): I5-20. Disponível em: http:// intjmi.com/article- I-347-en.html

17. Pimenta MVT, Macêdo SFM, Reis AS, Moura JRA, Oliveira ESO, Silva ARV. Condições de trabalho e qualidade de vida de trabalhadores da limpeza pública. Rev enferm UFPI [internet]. 2018 [acesso em 2 jul 2020]; 7(I):26-32. Disponível em: https://revistas.ufpi.br/index.php/reufpi/article/ view/6284/pdf

18. Portela NLC, Pedrosa AO, Silva NS, Ramos JS, Silva RS, San- 
tos RTC. Riscos ocupacionais entre trabalhadores de um serviço de atendimento móvel de urgência. ReonFacema [internet]. 2018[acesso em 2 jul 2020]; 4(2): 986-992. Disponível em: https://www.facema.edu.br/ojs/index.php/ReOnFacema/article/view/383/245

19. Salvi A, Patki G, Liu H, Salim S. Psychological Impact of Vehicle Exhaust Exposure: Insights from na Animal Model. Sci Rep, Houston Rep[internet]. 2017 [acesso em 2 jul 2020]; 7(8306). Disponível em: https://www.nature.com/articles/ s4I598-0I7-08859-I.pdf

20. Sedrez JA, Kasten APS, Chaise FO, Candotti CT. Fatores de risco para doenças cardiovasculares e osteomusculares relacionadas ao trabalho em profissionais do atendimento pré-hospitalar de urgência: uma revisão sistemática. Rev Bras Med Trab. [internet]. 2017 [acesso em 6 jul 2020];
I5(4): 355-63. Disponível em: https://cdn.publisher.gn I.link/ rbmt.org.br/pdf/vI5n4al I.pdf.

21. Mahdizadeh M, Jaberi AA, Bonabi TN. Massage Therapy in Management of Occupational Stress in Emergency Medical Services Staffs: a Randomized Controlled Trial. Int J Ther Massage Bodywork[internet]. 2019 [acesso em 6 jul 2020]; I2(I): 16-22. Disponível em: https://www.ncbi.nlm.nih.gov/ pmc/articles/PMC6398989/pdf/ijtmb-12-I6.pdf.

22. Canesin D, Lovadini V, Sakamoto S. As dificuldades vivenciadas pelos profissionais de enfermagem no atendimento pré -hospitalar. Revista Enfermagem Atual In Derme [internet]. 2020 [acesso em 2 set 2020]; 9 I(29): II0-17.

Recebido: 2020-07-18

Aceito: 2020-09-03 